\title{
Coledocolitiasis y exploración laparoscópica de la vía biliar. Un estudio de cohorte
}

\author{
Choledocholithiasis and laparoscopic exploration of the bile duct. \\ A cohort study
}

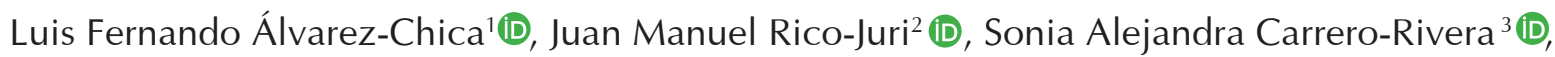 \\ Felipe Castro-Villegas ${ }^{3}$ D
}

1 Médico, especialista en Cirugía general, Cirugía laparoscópica avanzada y Gastroenterología clínico quirúrgica, Centro médico Imbanaco, Cali, Colombia.

2 Médico, especialista en Cirugía general y Cirugía hepato biliar y de trasplantes, Centro médico Imbanaco, Cali, Colombia.

3 Médico, especialista en Cirugía general y Cirugía de trasplantes, Centro médico Imbanaco, Cali, Colombia.

\section{Resumen}

Introducción. La coledocolitiasis es la presencia de cálculos en las vías biliares. En la mayoría de los casos se trata mediante la colangio pancreatografía retrógrada endoscópica y menos comúnmente por intervención quirúrgica laparoscópica. El objetivo de este estudio fue describir una cohorte retrospectiva de pacientes sometidos a exploración laparoscópica de la vía biliar.

Métodos. Se incluyeron pacientes intervenidos entre los años 2014 y 2018, en dos instituciones de nivel III en Cali, Colombia, referidos para valoración por cirugía hepato-biliar, por dificultad para la extracción de los cálculos por colangio pancreatografia retrograda endoscópica, debido al tamaño, la cantidad, o la dificultad para identificar o canular la papila duodenal.

Resultados. De los 100 pacientes incluidos, se encontró que el 72 \% fueron mujeres, con rango de edad entre 14 y 92 años. Al $39 \%$ de los pacientes se les extrajo un solo cálculo y al $16 \% 10$ cálculos. Un $12 \%$ presentaron cálculos gigantes (mayores de 2,5 cm de ancho) y un $44 \%$ presentaron litiasis múltiple. Al $69 \%$ de los pacientes se les realizó colecistectomía. El porcentaje de éxito de limpieza de la vía biliar por laparoscopia fue del 95 \%.

Discusión. La exploración laparoscópica de la vía biliar es una técnica posible, reproducible, segura y con excelentes resultados para el manejo de la coledocolitiasis.

Palabras clave: conducto colédoco; coledocolitiasis; procedimientos quirúrgicos mínimamente invasivos; laparoscopía; coledocoscopia; colangiopancreatografia retrógrada endoscópica.

Fecha de recibido: 20/04/2020 - Fecha de aceptación: 10/11/2020 - Fecha de publicación en línea: 05/03/2021

Correspondencia: Luis Fernando Álvarez Chica, Carrera 38 A No 5 A - 100, Cali, Colombia. Teléfono 3006174028

Correo electrónico: Ifalvarez@imbanaco.com.co

Citar como: Álvarez-Chica LF, Rico-Juri JM, Carrero-Rivera SA, Castro-Villegas F. Coledocolitiasis y exploración laparoscópica de la vía biliar. Un estudio de cohorte. Rev Colomb Cir. 2021;36:301-11. https://doi.org/10.30944/20117582.558

Este es un artículo de acceso abierto bajo una Licencia Creative Commons - BY-NC-ND https://creativecommons.org/licenses/by-ncnd/4.0/deed.es 


\begin{abstract}
Introduction. Choledocholithiasis is the presence of stones in the bile ducts. In most cases it is treated by endoscopic retrograde cholangio pancreatography and less commonly by laparoscopic surgery. The objective of this study was to describe a retrospective cohort of patients who underwent laparoscopic exploration of the bile duct.
\end{abstract}

Methods. The study included patients operated between 2014 and 2018, in two level III institutions in Cali, Colombia, referred for evaluation for hepato-biliary surgery, due to difficulty in removing stones by endoscopic retrograde pancreatography cholangiography, due to the size, quantity, or difficulty of identifying or cannulating the duodenal papilla.

Results. Out of the 100 patients included, it was found that $72 \%$ were women, with an age range between 14 and 92 years. A single stone was removed from $39 \%$ of patients and 10 stones from $16 \% ; 12 \%$ had giant stones (greater than $2.5 \mathrm{~cm}$ wide), and $44 \%$ had multiple stones; $69 \%$ of the patients underwent cholecystectomy. Laparoscopic bile duct cleaning success rate was $95 \%$.

Discussion. Laparoscopic exploration of the bile duct is a possible, reproducible, and a safe technique with excellent results for the management of choledocholithiasis.

Keywords: common bile duct; choledocholithiasis; minimally invasive surgical procedures; laparoscopy; choledochoscopy; cholangiopancreatography endoscopic retrograde.

\section{Introducción}

La incidencia de colelitiasis en la población adulta fluctúa entre el $6 \%$ y el $10 \%$ y de estos entre el $3 \%$ y el $16 \%$ presentan cálculos en sus vías biliares ${ }^{1-3}$. La laparoscopia es la técnica recomendada para el manejo de la enfermedad litiásica vesicular, pero el manejo de la coledocolitiasis aún es motivo de controversia ${ }^{4-7}$. Con la invención y el desarrollo de la colangio pancreatografia retrograda endoscópica (CPRE) y la esfinterotomía endoscópica (EE), se popularizó el manejo endoscópico prequirúrgico de la coledocolitiasis, seguido en un segundo tiempo (idealmente no más de 24 horas), por la colecistectomía laparoscópica (CL) ${ }^{8-10}$.

De igual forma, se han refinado y consolidado las técnicas para la exploración laparoscópica de la vía biliar (ELVB), pero son pocos los estudios que han comparado el manejo en un sólo tiempo quirúrgico contra el manejo secuencial. Los resultados de los estudios muestran que las dos modalidades son similares en términos de limpieza del colédoco y complicaciones, pero la estancia hospitalaria es más corta y el manejo en un solo tiempo resulta ser más económico ${ }^{11-13}$.

Recientemente se han publicado algunos metaanálisis que informan la superioridad del manejo de la colecisto-coledocolitiasis en un sólo tiempo. Dichos estudios no sólo resaltan la superioridad en términos de menor estancia hospitalaria y costo efectividad, sino también en términos de eficacia en la limpieza y seguridad posoperatoria, por lo que los autores consideran que el tratamiento en un solo tiempo quirúrgico es el óptimo o de elección. Sin embargo, se debe contar con el respaldo institucional, la disponibilidad tecnológica y el recurso humano entrenado para la realización de dicho procedimiento ${ }^{14-16}$.

El objetivo del presente estudio fue analizar una cohorte retrospectiva de pacientes llevados a ELVB entre los años 2014 y 2018, en dos instituciones de nivel III en la ciudad de Cali, Colombia.

\section{Métodos}

Estudio descriptivo de cohorte histórico en el que se incluyeron 100 pacientes que fueron llevados a cirugía para ELVB, entre septiembre de 2014 y diciembre de 2018, con diagnóstico de coledocolitiasis confirmado mediante colangio resonancia magnética nuclear (CRMN) o de CPRE. La mayoría de ellos (89\%), fueron remitidos desde el servicio de gastroenterología para valoración por cirugía hepato biliar después de una CPRE fallida, por 
múltiples cálculos o cálculos de gran tamaño, después de haber practicado papilotomía o colocación de prótesis biliares. Un menor número de pacientes $(11 \%)$ fueron diagnosticados mediante CRMN y llevados a tratamiento quirúrgico laparoscópico en un solo tiempo.

\section{Técnica quirúrgica}

Todos los pacientes se intervinieron con técnica laparoscópica convencional utilizando cuatro trocares, teniendo cuidado de ubicar el cuarto trocar (de $5 \mathrm{~mm}$ y de material plástico) en la línea medio clavicular, inmediatamente debajo de la costilla y en forma perpendicular a la unión cístico-vesicular, para facilitar la introducción del coledocoscopio. Varios pacientes tenían el antecedente de colecistectomía, con presencia de adherencias del epiplón al lecho hepático, lo que dificultó el acceso hasta la vía biliar (VB) extra hepática, por lo cual fue necesario usar el disector armónico.

Una vez identificada la VB extra hepática, se introdujo por el puerto epigástrico una aguja de tuberculina de calibre 26 y con la ayuda del disector de Maryland se realizó la punción de la VB, comprobando que se trataba de ella al ver fluir material bilioso por la aguja. Inmediatamente, se retiró el trocar epigástrico de $10 \mathrm{~mm}$ y a través de la incisión cutánea se introdujo un bisturí No 11 de mango largo con el cual se incidió longitudinalmente el colédoco en su parte más anterior, logrando un corte nítido y preciso, de un tamaño proporcional al tamaño de los cálculos, ya conocidos por las imágenes de la CRMN y por la visión directa del colédoco dilatado y de los cálculos.

$\mathrm{Al}$ seccionar el colédoco se observó cómo algunos cálculos fluyeron; utilizando dos disectores de "grasper" y compresión ascendente suave, se facilitó la salida de los cálculos más superficiales. Esta maniobra también se realizó por encima del duodeno, tratando de llevar hasta la incisión en el colédoco los cálculos más distales. Al no tener más cálculos visibles, se introdujo el coledocoscopio por el trocar subcostal, inicialmente hacia proximal y luego hacia distal de la VB.

Bien sea con técnica transcística o en exploración directa, algunas veces se encontraron cálculos impactados en la papila duodenal, los cuales pudieron ser empujados hasta el duodeno distal con el coledocoscopio o con solución salina (SS) inyectada a presión. Para ello, se pidió al anestesiólogo que suspendiera cualquier derivado opiáceo que causara espasmo del esfínter de Oddi y que administrara al paciente hioscina para lograr dilatarlo (idealmente se debe usar glucagón, pero no es fácil disponer de él). En la mayoría de las exploraciones biliares se puede, sin forzar demasiado la papila, realizar dicha maniobra, ver las vellosidades intestinales y aún más, ver los cálculos flotando en el duodeno (video 1, https://www.youtube.com/watch?v=jK_p0eQhZRk\&feature=youtu.be\&ab_channel=LuisFernandoAlvarez Cálculo "flotando" en el duodeno. https://www.youtube.com/watch?v=xzt3PL5BfQg+-+_JU9rQOivbI+\%E2\%80\%93+nAlScXqtXpY+\%E2\%80\%93+3M3kLHx-prc\&feature=youtu.be Exploración laparoscópica directa de la vía biliar). Incluso, se puede apreciar la papila en forma anterógrada, diagnosticar alguna alteración y obtener material para biopsia. No se presentaron casos de pancreatitis aguda por esta maniobra.

Si se siguen encontrando cálculos en el colédoco, se usa irrigación con solución salina introduciendo una sonda de Nélaton por el trocar subcostal y llevándola hasta el colédoco distal para producir turbulencia y lograr que los cálculos migren hacia la coledocotomía. Si esta maniobra no funciona, se introduce una canastilla de "Dormia" con la cual se logran extraer generalmente todos los cálculos, con o sin visión directa, dejando finalmente la VB limpia y permeable. Actualmente se dispone de dos coledocoscopios, uno de 2,2 $\mathrm{mm}$ para uso transcístico en aquellos casos de cálculos pequeños (menores de $8 \mathrm{~mm}$ ) y otro de $5 \mathrm{~mm}$ que se utiliza en la exploración directa de la vía biliar (Figura 1).

Cuando la exploración y extracción transcística de cálculos es exitosa, el curso de la cirugía es similar al de una colecistectomía simple, es decir, su manejo es ambulatorio. En la experiencia inicial siempre se dejaba un tubo en T, pero luego se comenzó a hacer uso de una sutura primaria con puntos separados de Vicryl ${ }^{\circledR}$, y actualmente se 


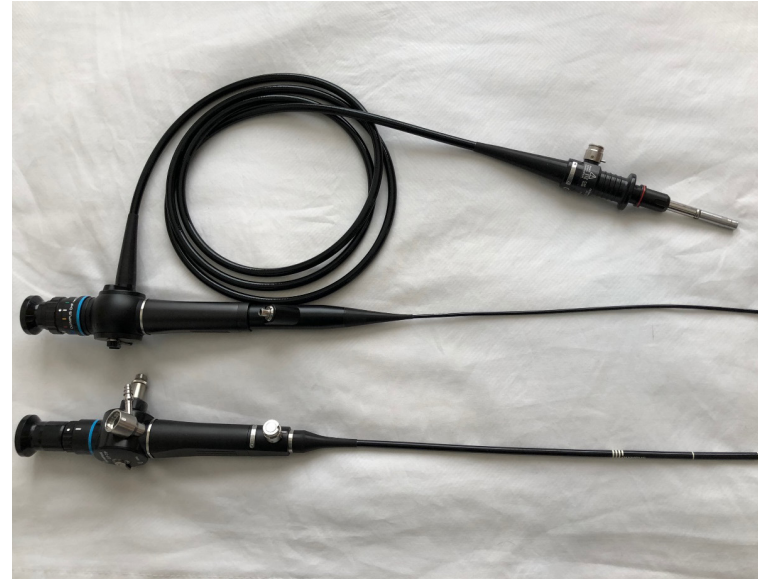

Figura 1. Coledocoscopios de $2.2 \mathrm{~mm}$ y $5.0 \mathrm{~mm}$

realiza una sutura corrida de PDS ${ }^{\circledR}$ 4-0. En casos de exploración directa y coledocotomía, se deja de rutina y por máximo 48 horas un drenaje subhepático.

Algunos pacientes llegaron con stents plásticos en la VB, los cuales fueron retirados para hacer la exploración biliar y el retiro de los cálculos. Dichas prótesis no fueron reinsertadas. Se prefirió dejar un drenaje subhepático como se mencionó anteriormente.

\section{Video coledocoscopia}

La coledocoscopia se refiere a la utilización de endoscopios convencionales delgados y flexibles (2-5 mm) durante una ELVB. Los aparatos se introducen por uno de los trocares percutáneos (usualmente por el trocar subcostal medio clavicular), y tienen por finalidad, la extracción de cálculos de la VB ${ }^{17-20}$, como fue el objetivo de este estudio.

\section{Video colangioscopia}

En la colangioscopia se utiliza un colangioendoscopio delgado y largo, con un diámetro externo de 3,2 mm y un canal de trabajo de 1,2 $\mathrm{mm}$, que es introducido por el canal de trabajo de un duodenoscopio jumbo (canal de trabajo de $4,2 \mathrm{~mm}$ ), el cual se posiciona frente a la papila duodenal y una vez hecha la EE, permite avanzar el colangio-endoscopio dentro de los conductos biliares. Este sistema es llamado comúnmente "mother-baby" y sus principales desventajas son el alto costo, la fragilidad del equipo y la necesidad de un segundo endoscopista para su operación ${ }^{21}$.

Estas razones favorecieron el desarrollo del sistema Spy Glass ${ }^{\circledR}$ el cual es un colangioscopio de un solo operador, que es insertado por el canal de trabajo del duodenoscopio y consiste en un catéter de 10 Fr y $230 \mathrm{~cm}$ de largo, que a su vez permite el paso de una fibra óptica reutilizable de $0,9 \mathrm{~mm}$ de diámetro. El catéter óptico provee una imagen de 6000 pixeles y maniobrabilidad en la punta en cuatro direcciones, con un ángulo de 30 grados en cada dirección. El sistema permite irrigación permanente con solución salina para lograr mantener claridad en la imagen ${ }^{22,23}$.

El último desarrollo es el sistema digital Spy Glass ${ }^{\circledR}$ DS que provee aún mayor resolución en la imagen, mayor amplitud de visión con fuente de luz integrada y unidad de cámara ${ }^{24}$. Actualmente ya está disponible el Spy Glass ${ }^{\circledR}$ DS II. Un método alternativo de colangioscopia peroral, que consiste en el uso de un delgado endoscopio de $5 \mathrm{~mm}$ de diámetro que se pasa por la nariz y se ubica frente a la papila duodenal, accediendo a la vía biliar mediante una EE, aunque algunas veces es difícil llegar, ubicar y lograr penetrar la papila y frecuentemente se hacen asas en el estómago o el duodeno.

La principal utilidad de la colangioscopia peroral está en el diagnóstico de estenosis indeterminadas del árbol biliar, de lesiones ampulares y su extensión dentro del árbol biliar, estadiaje de colangiocarcinoma, evaluación de lesiones quísticas de las vías biliares, estudio de la hemobília, de defectos de llenado inexplorados, evaluación de complicaciones biliares postrasplante, así como la posibilidad de tomar biopsias dirigidas ${ }^{24}$.

La colangioscopia también tiene utilidad terapéutica, permitiendo realizar litotrípsia (electro hidráulica o láser) en cálculos gigantes o de difícil extracción, retiro de prótesis pancreáticas migradas, terapia de ablación en tumores (argón plas$\mathrm{ma}$ ), retiro de parásitos (áscaris, fasciola), drenaje trans papilar de la vesícula, manejo de cálculos y estenosis del conducto pancreático ${ }^{25}$. 


\section{Resultados}

La mayoría de los pacientes fueron mujeres (72\%), con un rango de edad entre 14 años y 92 años. El $70 \%$ de los pacientes estaba entre 30 años y 70 años, con un pico máximo de $21 \%$ entre 60 años y 70 años. La máxima cantidad de cálculos extraídos en un solo paciente fueron 16; al $39 \%$ de pacientes se les extrajo un solo cálculo y al $16 \%$ de pacientes se les extrajeron 10 cálculos. Se encontraron 12 pacientes con cálculos gigantes mayores de 2,5 cm de ancho, de los cuales 6 presentaron moldes de cálculos con 5 o más $\mathrm{cm}$ de largo (figura 2).

Llama la atención la alta frecuencia de pacientes $(n=44)$ con litiasis múltiple (tres cálculos o más). En cuatro pacientes con cálculos de $3 \mathrm{~cm} \mathrm{o}$ más, litiasis múltiple, litiasis recidivante y ausencia de vesícula, se realizó una derivación bilio-digestiva, específicamente una hepático-yeyunostomía en Y de Roux por laparoscopia, con sección del yeyuno con endograpadora lineal cortante y carga blanca $(2,5 \mathrm{~mm})$ a $70 \mathrm{~cm}$ distal al ángulo de Treitz, la anastomosis yeyuno-yeyunal a $80 \mathrm{~cm}$ con endograpadora lineal cortante y carga blanca, y la anastomosis hepático-yeyunal con puntos separados de $\mathrm{PDS}^{\circledR}$ 4-0.

De los 100 pacientes del estudio, 31 tenían colecistectomía previa, en los restantes 69 se realizó la colecistectomía y exploración de la vía biliar. A 14 pacientes se les realizó colangiografía intraoperatoria (CIO), a ocho por duda diagnóstica previa a la cirugía y a seis por duda en la limpieza de la VB.

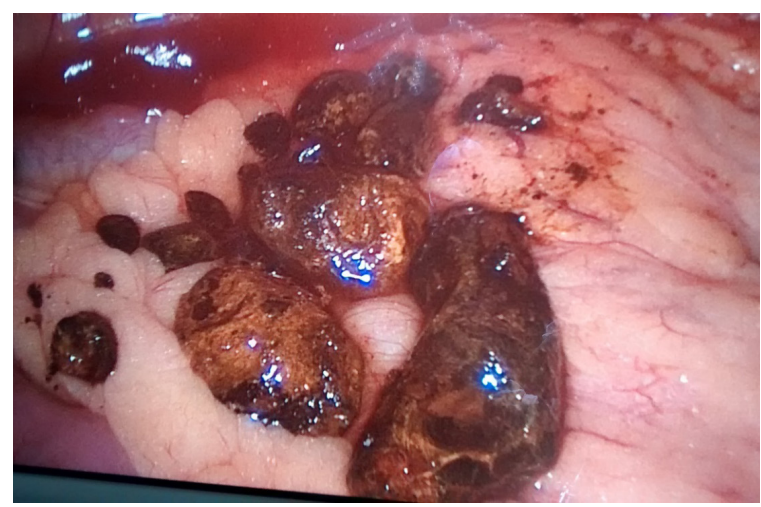

Figura 2. Moldes de cálculos gigantes hallados en la exploración laparoscópica directa.
Se realizó exploración directa en 67 pacientes (video 1), abriendo longitudinalmente el colédoco con bisturí de punta fina, y en 32 se practicó una exploración transcística (video 2, https://www.youtube.com/ watch?v=w4RcTAirwhg+-+JEU3n42Zydw+-+yZJAWLSrF3A+-+++++wqcAm0CLij8++-++2KR7dq0epbA\&feature=youtu.be Exploraciónlaparoscópica transcística de la vía biliar).

En un paciente se usó la técnica mixta (combinación de las dos anteriores), (video 3, https://youtu. be/WnHtHyaG9lA Exploración laparoscópica con técnica mixta: transcística y directa). En cuanto a la forma de suturar la vía biliar, en 53 pacientes se realizó sutura corrida con PDS ${ }^{\circledR} 4-0$ (video 4, https:// youtu.be/zKUy-JdgHec Sutura continua del colédoco), en 39 pacientes puntos separados y en ocho, tubo en $\mathrm{T}$ (técnica usada al inicio de la experiencia).

Cuando se practicó colecistectomía, el cístico se ligó en 64 pacientes con clips de titanio LT 400, en tres pacientes se realizó ligadura con puntos separados y en dos de ellos rafia con endograpadora lineal cortante y carga verde $(4,8 \mathrm{~mm})$ debido al grosor e inflamación de sus paredes. A tres pacientes con esfinterotomía previa y cálculos en el colédoco distal parcialmente impactados, se le empujaron los cálculos hasta el duodeno (video 5, https://youtu.be/W1X-7jYXoZo Avance del cálculo impactado al duodeno). Un solo paciente presentó pancreatitis leve que se resolvió con manejo médico. En 12 pacientes se retiró la prótesis previamente insertada por el endoscopista, al no poder drenar completamente la VB debido al número o al tamaño de los cálculos (video 6, https://www.youtube.com/watch?v=F-WR2p9w_ko Extracción de prótesis insertada en el colédoco).

Se tuvo éxito del $95 \%$ en la extracción y limpieza de la VB por laparoscopia, porcentaje que hubiese podido ser mayor, con la disposición de mejor tecnología como la litotrípsia láser o electrohidráulica. En seis pacientes se encontró litiasis intrahepática diagnosticada al introducir el coledocoscopio, la cual fue resuelta satisfactoriamente en cinco pacientes. Un paciente requirió re-exploración abierta en un segundo tiempo, debido a litiasis residual detectada en el control postquirúrgico del primer mes, al consultar por dolor abdominal e ictericia leve y en quien la CRMN mostró un cálculo en el hepático izquierdo. 
A un paciente tratado por litiasis múltiple, se le practicó CPRE posquirúrgica, debido a duda en la limpieza completa de la VB, encontrando un cálculo residual el cual fue extraído. Durante la exploración laparoscópica del colédoco, en un paciente se encontró una fístula colecisto duodenal, la cual fue tratada seccionándola con endo grapadora lineal cortante con carga blanca.

En el $5 \%$ de los pacientes se realizó conversión a cirugía abierta, dos por adherencias firmes que impidieron el acceso al abdomen, dos por cálculos impactados distales que no pudieron removerse con técnica laparoscópica y un paciente por un cálculo en el conducto hepático izquierdo que no pudo extraerse con canastilla. A todos los pacientes se les realizó un seguimiento a los 15 días, 1, 3 y 6 meses, detectándose litiasis residual en tres pacientes, la cual fue diagnosticada con CRMN y resuelta en dos pacientes con CPRE y en uno con cirugía abierta (litiasis intrahepática).

\section{Duración de cirugía}

El promedio del tiempo quirúrgico fue de $160 \mathrm{mi}-$ nutos, con un tiempo mínimo de 75 minutos en una paciente de colecistectomía, y exploración transcística con un solo cálculo. El tiempo máximo fue de 210 minutos, en un paciente con colecistectomía abierta previa y múltiples adherencias, en quien la mayoría del tiempo (más de una hora), se empleó en la disección, hasta lograr el abordaje del colédoco, y por la litiasis múltiple, necesito varias coledocoscopias para comprobar la limpieza de la VB.

\section{Posoperatorio}

En cuanto a la estancia intrahospitalaria, el promedio fue de tres días. En dos pacientes se hizo manejo ambulatorio, luego de colecistectomía y exploración transcística con una muy fácil extracción del cálculo. Cuatro pacientes con exploración transcística solo requirieron hospitalización por dos días. El tiempo máximo fue de 15 días, en una paciente de baja escolaridad, residente en un área rural lejana y en quien se encontró una fistula biliar.

\section{Complicaciones}

Se presentaron tres complicaciones grado I de acuerdo con la clasificación de Clavien-Dindo ${ }^{26}$. Una paciente con fístula biliar a la cual se le practicó cierre con sutura corrida en la cirugía inicial, y que requirió sonda de drenaje por 15 días más. Un paciente presentó pancreatitis leve como consecuencia del repetido paso de la canastilla de Dormia hacia distal, y mejoró con el manejo médico, y un tercer paciente que presentó un hematoma a nivel del puerto umbilical, que requirió drenaje con anestesia local sin afectar su curso posoperatorio. No hubo mortalidad en esta serie de casos.

\section{Discusión}

En 1882 Karl August Langenbuch, describió la exploración de la vía biliar. Por más de 100 años, al encontrar coledocolitiasis o alguna indicación para explorar la VB durante una colecistectomía abierta, esta era realizada por el cirujano. Desde 1974 con la invención de la CPRE y la EE por Kawai y Classen, se prefirió hacer en un primer tiempo endoscópico el diagnóstico y de ser el caso, la extracción de los cálculos, y en un segundo tiempo la

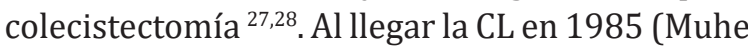
en Alemania), tomó mucha más fuerza la CPRE y la EE, debido a la imposibilidad de realizar la exploración de la VB por la vía laparoscópica ${ }^{29}$, pero bastaron pocos años para que unos cuantos cirujanos (Berci, Petelin, Phillips, Perisat, entre otros), iniciaran la ELVB ${ }^{30-32}$, la cual ha tenido importante desarrollo y actualmente es una técnica completamente aceptada, tanto como la CPRE.

Hoy en día, si durante una CL hay indicación de explorar la VB, o si se conoce previamente la concomitancia de colelitiasis y coledocolitiasis, queda a voluntad del cirujano, según su capacidad, nivel de experticia y el recurso tecnológico de la institución, elegir cuál de las dos vías es la más adecuada para resolver el problema de su paciente ${ }^{33}$.

En general todos los hospitales o instituciones de salud de tercer nivel, cuentan con cirujanos y gastroenterólogos capacitados para tratar el problema de la litiasis coledociana por diferentes vías. Los primeros con técnica de mínima invasión (ELVB) y en un solo tiempo y los segundos mediante la CPRE pre o posoperatoria. Con cualquiera de las dos técnicas se puede lograr el objetivo, pero si el paciente también requiere colecistectomía, no hay duda de que hacerlo todo en un solo tiempo quirúrgico, es más costo efectivo para el paciente. 
Se podría decir entonces que "todos los caminos conducen a Roma”, pues antes, o después, o de una vez, siempre se pretende llegar al mismo objetivo que es dejar la VB libre de cálculos.

En las instituciones donde se desarrolló esta investigación, se cuenta con equipos de alta tecnología, con endoscopistas idóneos en la realización de técnicas invasivas de la VB, con un grupo de cirujanos entrenados en técnicas de cirugía laparoscópica avanzada y con experticia en ELVB, y además se cuenta con un grupo de cirujanos hepato-bilio pancreáticos. La VB siempre fue y ha sido una estructura manejada por el cirujano, que con la llegada de la CPRE se le prestó o entregó al clínico, pero ahora, con el desarrollo de la ELVB, el cirujano reclama que se le devuelva un órgano que históricamente ha sido suyo.

Desde los primeros reportes de la ELVB en $1991^{34}$, ésta ha ido ganando aceptación y cada vez aparecen más estudios y metaanálisis demostrando sus ventajas sobre la técnica en dos

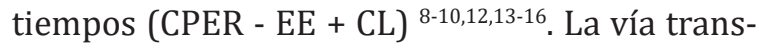
cística es la ideal, pero esto depende de algunas consideraciones anatómicas como el tamaño del cístico, distorsión, obstrucción, inflamación o estenosis del cístico, alteraciones de las válvulas de Heister, variantes anatómicas en la implantación del conducto cístico o adherencias firmes en la unión del cístico con la VB común, además del tamaño, número, localización y consistencia de los cálculos. Cuando se hace exploración transcística, su curso es similar al de una colecistectomía con mínimo trauma, rápida recuperación y corta hospitalización, muchas veces también ambulatoria, como una colecistectomía simple ${ }^{35-37}$.

De no ser posible la vía transcística, la coledocotomía directa ofrece la posibilidad de extraer cálculos de mayor tamaño, acceder al hepático común y a la VB intrahepática ${ }^{38-40}$, además, si es necesario, de una vez efectuar derivaciones bilio digestivas. En este estudio, el mayor número de pacientes $(n=89)$, fueron remitidos por el gastroenterólogo al no poder retirar cálculos de gran tamaño por CPRE, lo cual es relativamente sencillo para los cirujanos vía laparoscópica por coledocotomía directa, la cual permite además retirar las prótesis biliares. El coledocoscopio se puede introducir fácilmente por el puerto subxifoideo de $5 \mathrm{~mm}$ para corroborar el número y el tamaño de los cálculos y acceder a la vía biliar intrahepática, verificando la ausencia de cálculos, y procediendo a la extracción y limpieza de la VB ${ }^{41,42}$.

En algunas ocasiones se efectúa coledocoscopia a pacientes con estenosis de las anastomosis bilio digestivas, sometidos a procedimientos abiertos, y en quienes, durante la reintervención, los cirujanos hepatobiliares quieren estar seguros de que no queden cálculos proximales a la nueva anastomosis, lo que más tarde puede generar complicaciones e incluso colangitis, inflamación crónica o una nueva estenosis ${ }^{43}$.

La fístula biliar se define como la presencia de material líquido a través del drenaje subhepático, con un incremento en la concentración de bilirrubinas, al menos tres veces superior al de los niveles hemáticos, o como una colección de material biliar después del tercer día posoperatorio, que requiere reintervención o drenaje por radiología ${ }^{44}$. En esta serie de pacientes, sólo se presentó un caso de fístula biliar (Grado A), comparable con el estudio de Gómez et al. ${ }^{45}$ donde se presentó sólo un paciente con fuga biliar.

La litotrípsia láser se puede realizar idealmente con láser neodimio-YAG y existen múltiples reportes que la presentan como una técnica eficaz y segura en el manejo de cálculos impactados en la papila. La fibra láser se avanza por el canal de trabajo del coledocoscopio ${ }^{46-48}$. Actualmente esta técnica no se utiliza en la institución donde se realizó el presente estudio, pero se considera que vale la pena explorarla. De igual forma, tampoco se emplea la CPRE intraoperatoria, pero se conoce de su importancia y utilidad en otras instituciones, donde se usa principalmente en casos de cálculos impactos distales ${ }^{49,50}$.

La hepático-yeyunostomía está indica en pacientes con colédoco de $3 \mathrm{~cm}$ o más, litiasis voluminosa y múltiple, incluso intrahepática, litiasis recidivante y ausencia de vesícula, casos en los que también se puede realizar la hepático-duodenostomía. Esto permite un tratamiento completo y definitivo, principalmente en pacientes ancianos. De acuerdo con Lechaux, en esta anastomosis termino-lateral, para la exclusión yeyunal se emplea el método del asa en Y que implica la sustitución de la vía biliar, y se tienen algunas variantes a esta 
técnica como la confección de un asa yeyunal en Y por sutura automática ${ }^{51}$.

En el estudio de Concepción-Quiñones et al. ${ }^{52}$, realizado entre 2007 y 2011 en el Hospital Enrique Cabrera de La Habana, Cuba, los autores encontraron que la técnica de preferencia en las lesiones de vías biliares fue la hepático-yeyunostomía, seguida por la colédoco-yeyunostomía y la colédoco-duodenostomía. De hecho, Hirano et $a l .{ }^{53}$, después de abordar diferentes técnicas de reconstrucción biliar, encontraron que la hepático-yeyunostomía es un procedimiento seguro, factible y puede ser una buena opción para la calidad de vida en personas con enfermedad maligna hepatobiliar. Además, otros autores han resaltado su preferencia por este procedimiento, debido a su corto tiempo quirúrgico, en comparación con otros procedimientos, y a que minimiza el riesgo de complicaciones ${ }^{54}$.

\section{Conclusión}

Con los resultados obtenidos en este trabajo, se contribuye a demostrar que la ELVB es posible, segura y con excelentes resultados. Aunque por diferentes caminos se puede llegar a la VB, y tratarla, se recomienda e incentiva a los cirujanos generales para que, de nuevo recuperen un área de trabajo, que desde antaño siempre nos ha correspondido. Se propone un flujograma para el manejo de pacientes con sospecha de coledocolitiasis (Figura 3).

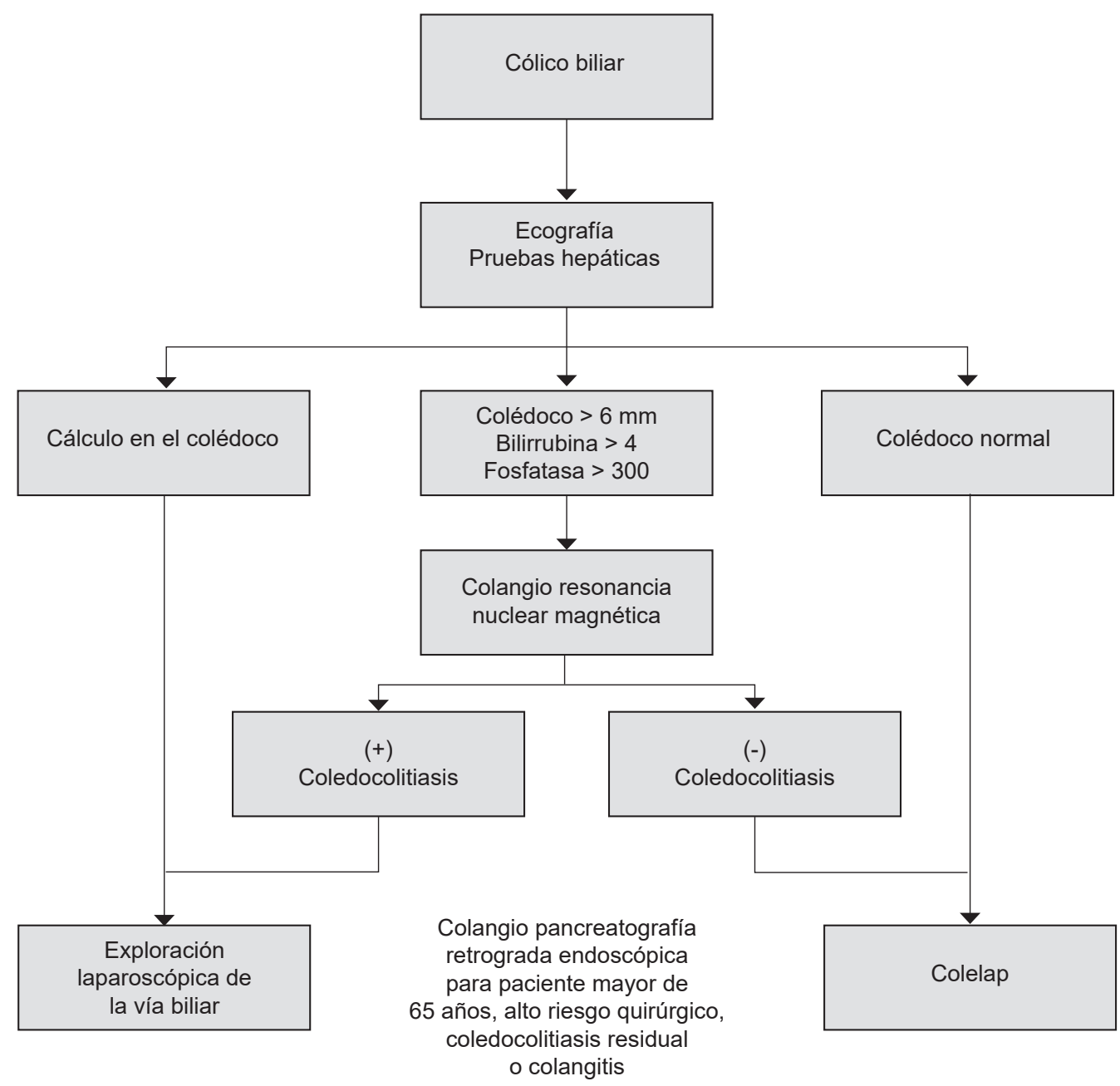

Figura 3. Flujograma para el manejo de pacientes con sospecha de coledocolitiasis 


\section{Cumplimiento de normas ética}

Consentimiento informado: Este estudio es una revisión retrospectiva de historias clínicas, y como tal, no requiere de un consentimiento informado

Conflicto de interés: Ninguno reportado por los autores

Fuentes de financiación: Este estudio fue financiado con recursos propios de los autores.

\section{Contribución de los autores:}

Concepción y diseño del estudio: Luis Fernando ÁlvarezChica, Juan Manuel Rico-Juri, Sonia Alejandra CarreroRivera, Felipe Castro-Villegas.

Adquisición, análisis e interpretación de datos: Luis Fernando Álvarez-Chica, Juan Manuel Rico-Juri, Sonia Alejandra Carrero-Rivera, Felipe Castro-Villegas.

Redacción del manuscrito: Luis Fernando Álvarez-Chica, Juan Manuel Rico-Juri, Sonia Alejandra Carrero-Rivera, Felipe Castro-Villegas.

Revisión crítica: Luis Fernando Álvarez-Chica, Juan Manuel Rico-Juri, Sonia Alejandra Carrero-Rivera, Felipe Castro-Villegas.

\section{Referencias}

1. Barreras González JE, Torres Peña R, Ruiz Torres J, Martínez Alfonso MA, Roque González R, Brizuela Quintanilla R. Algoritmo para el tratamiento mínimamente invasivo de la coledocolitiasis. Rev Cuba Cir. 2014;53:346-55.

2. Stoker ME, Levenecc RJ, McCann JC. Laparoscopic common bile duct exploration. J Laparoendosc Surg. 1991;1:287-93. https://doi.org/10.1089/lps.1991.1.287

3. Jinfeng Z, Yin Y, Chi Z, Junye G. Management of impacted common bile duct stone during a laparoscopic procedure: A retrospective cohort study of 377 consecutive patient. Int J Surg. 2016;32:1-5. https://doi.org/10.1016/j.ijsu.2016.06.006

4. Phillis E, Berci G, Barker K, Williams J. The role of choledochoscopy: The eternal problem of how to remove a CBD stone. Surg Innov. 2015;22:540-5. https://doi.org/10.1177/1553350615594444

5. Cuschieri A, Lezoche E, Morino M, Croce E, Lacy A, Toouli J, et al. E.A.E.S. multicenter prospective randomized trial comparing two-stage vs single-stage management of patients with gallstone disease and ductal calculi. Surg Endosc. 1999;13:952-7. https://doi.org/10.1007/s004649901145

6. Berci G, Morgensten L. Laparoscopic management of common bile duct stones. A multi-institucional SAGES study. Surg Endosc. 1994;8:1168-75. https://doi.org/10.1007/BF00591044
7. Lezoche E, Paganini AM. Single stage laparoscopic treatment of gallstones and common bile duct stones in 120 unselect consecutive patients. Surg Endosc. 1995;9:1070-5. https://doi.org/10.1007/BF00188989

8. Zhu HY, Xu M, Shen HJ, Yang C, Li F, Li KW, et al. A Meta-analysis of single stage vs two stage management for concomitant gallstones and common bile duct stones. Clin Res Hepatol Gastroenterol. 2015;39:584-93. https://doi.org/10.1016/j.clinre.2015.02.002

9. GaoYC, Chen J, Qin Q Chen H, Wang W, Zhao J, etal.Efficacy and safety of laparoscopic bile duct exploration vs endoscopic sphinterotomy for concomitant gallstones and common bile duct stones: A meta-analysis of randomized controlled trials. Medicine (Baltimore). 2017;96:e7925. https://doi.org/10.1097/MD.0000000000007925

10. Martin DJ, Vernon D, Toouli J. Surgical versus endoscopic treatment of bile duct stones. Cochrane Database Syst Rev. 200619;(2):CD003327. https://doi.org/10.1002/14651858

11. Ding GQ, Cai W, Qin MF. Single-State vs. Two-stage management for concomitant gallstones and common bile duct stones: A prospective randomized trial with longterm follow up. J Gastrintest Surg. 2014;18:947-51. https://doi.org/10.1007/s11605-014-2467-7

12. Liu JG, Wang YJ, Shu GM, Lou C, Zhang J, Du Z. Laparoscopic vs endoscopic management of choledocholithiasis in patients undergoing laparoscopic cholecystectomy: A meta-analysis. J Laparoendosc Adv Surg Teach. A 2014;24:287-94. https://doi.org/10.1089/lap.2013.0546

13. Mattila A, Mrena J, Kellokumpu I. Cost-analysis and effectiveness of on-stage laparoscopic versus two-stage endolaparoscopic management of cholecystocholedocholithiasis: A retrospective cohort study. BMC Surgery. 2017;17:79. https://doi.org/10.1186/s12893-017-0274-2

14. Lyu Y, Cheng Y, Li T, Cheng B, Jin X. Laparoscopic common bile duct exploration plus cholecystectomy versus endoscopic retrograde cholangiopancreatography plus laparoscopic cholecystectomy for cholecystocholedocholithiasis: A meta-analysis. Surg Endosc. 2019;33:3275-86. https://doi.org/10.1007/s00464-018-06613-w

15. Ricci C, Pagano N, Taffurelli G, Pacilio PA, Migliori M, Bazzoli $\mathrm{F}$ et al. Comparison of efficacy and safety of 4 combinations of laparoscopic and intraoperative techniques for management of gallstone disease with biliary duct calculi. A systematic review and network meta-analysis. JAMA Surgery. 2018;153:e181167 https://doi.org/10.1001/jamasurg.2018.1167

16. Pan L, Chen M, Ji L, Zheng L, Yan P, Fang J, et al. The safety and efficacy of laparoscopic common bile duct exploration combined with cholecystectomy for the management of cholecysto-choledocholithiasis. An upto-date meta-analysis. Ann Surg. 2018;268:247-53. https://doi.org/10.1097/SLA.0000000000002731 
17. Escat J, Gluksman DL, Maigne C, Fourtanier G, Fournier D, Valslic C. Choledochoscopy in surgery for choledocholithiasis. Am J Surg. 1984;147:670-1. https://doi.org/10.1016/0002-9610(84)90138-7

18. Motson RW, Wetter LA. Operative choledochoscopy: Common bile duct exploration is incomplete without it. Br J Surg. 1990;77:975-82. https://doi.org/10.1002/bjs.1800770907

19. Campagnacci R, Baldoni A, Baldarelli M, Rimini M, De Sanctis A, Di Emilio M. Is laparoscopic fiberoptic choledochoscopy for common bile duct stones a fine option or a mandatory step?. Surg Endosc. 2010;24:547-53. https://doi.org/10.1007/s00464-009-0599-8

20. Atamanalp SS, Yildirgan MI, Ozogul B, Ozturk G, Aydinli B, Kantarci M. Intraoperative flexible choledochoscopy: Outcomes of 216 cases over 23 years. Pak J Med Sci. 2012;28:83-6.

21. Ming-Ming Xu, Kahaleh M. Recent developments in choledochoscopy: Technical and clinical advances. Clin Exp Gastroenterol. 2016;9:119-24. https://doi.org/10.2147/CEG.S84020

22. Ramchandani M, Reddy DN, Lakhtakia S, Tandan M, Maydeo A, Chandrashekhar TS. Per oral cholangiopancreatoscopy in pancreatico biliary diseases - expert consensus statements. World J Gastroenterol. 2015;21:4722-34. https://doi.org/10.3748/wjg.v21.i15.4722

23. Karagyozov P, Boeva I, Tishkov I. Role of digital single-operator cholangioscopy in the diagnosis and treatment of biliary disorders. Word J Gastrointest Endosc. 2019;11:31-40. https://doi.org/10.4253/wjge.v11.i1.31

24. Pereira P, Peixoto A, Andrade P, Macedo G. Peroral cholangiopancreatoscopy with the spy glass system: What do we know 10 years later. J Gastrointestin Liver Dis. 2017;26:165-70.

https://doi.org/10.15403/jgld.2014.1121.262.cho

25. Chen YK, Parsi MA, Binmoeller KF. Single-operator cholangioscopy in patients requiring evaluation of bile duct disease or therapy of biliary stones (with videos). Gastrointest Endosc. 2011;74:805-14. https://doi.org/10.1016/j.gie.2011.04.016

26. Clavien PA, Barkun J, de Oliveira ML, Vauthey JN, Dindo $\mathrm{D}$, Schulick RD, et al. The Clavien-Dindo classification of surgical complications: five-year experience. Ann Surg. 2009;250:187-96. https://doi.org/10.1097/SLA.0b013e3181b13ca2

27. Kawai K, Akasaka Y, Murakami K. Endoscopic Sphincterotomy of the ampulla of Vater. Gastrointest Endosc. 1974;20:148. https://doi.org/10.1016/S0016-5107(74)73914-1

28. Classen M, Demling M. Endoskopische sphinteroctomy der papilla Vateri. Dtsch Med Wochenschr. 1974;99:4967. https://doi.org/10.1055/s-0028-1107790.
29. Ibáñez L, Escalona A, Devaud N, Montero P, Ramirez E, Pimentel F. Colecistectomía laparoscópica: Experiencia de 10 años en la Pontificia Universidad Católica de Chile. Rev Chil Cir. 2007;59:10-5. https://doi.org/10.4067/S0718-40262007000100004

30. Phillips EH, Carroll BJ, Pearltein AR. Laparoscopic choledochoscopy and extraction of common bile duct stones. World J Surg. 1993;17:22-8. https://doi.org/10.1007/BF01655700

31. Fletcher DR. Percutaneus (laparoscopic) cholecystectomy and exploration of the common bile duct: The common bile duct stone reclaimed for the surgeon. Aust NZJ Surg. 1991;61:814-5. https://doi.org/10.1111/j.1445-2197.1991.tb00161.x

32. Petelin JB. Laparoscopic common bile duct exploration. Surg Endosc. 2003;17:1705-15. https://doi.org/10.1007/s00464-002-8917-4

33. Palermo M, Duza G, Caviglia ML, De Innocentis N, Egan P, Fiscella G. Tratamiento de la litiasis coledociana mediante cirugía laparoscópica, endoscopía o combinado. Acta Gastroenterol Latinoam. 2015;45:90-6.

34. Petelin JB. Laparoscopic approach to common bile pathology. Surg Laparosc Endosc. 1991;1:33-41.

35. Gupta N. Role of laparoscopic common bile duct exploration in the management of choledocholithiasis. World J Gastrointest Surg. 2016;8:376-81. https://doi.org/10.4240/wjgs.v8.i5.376

36. Qandeel H, Zino S, Hanif Z, Nassar MK. Basket in catheter acces for transcystic laparoscopic bile duct exploration: Technique and results. Surg Endosc. 2016;30:1958-64. https://doi.org/10.1007/s00464-015-4421-5

37. Alvarez LF, Franco AL. Exploración laparoscópica del colédoco. Rev Colomb Cir. 1999;14:85-96.

38. Qui W, Sun XD, Wang GY, Zhang P, Du XH, Lv GY. The clinical efficacy of laparoscopy combined with choledochoscopy for cholelithiasis and choledocholithiasis. Eur Rev Med Pharmacol Sci. 2015;19:3649-54.

39. Vindal A, Chander J, Lal P, Mahendra B. Comparison between intraoperative cholangiography and choledochoscopy for ductal clearence in laparoscopic cbd exploration: A prospective randomized study. Surg Endosc. 2015;29:1030-38. https://doi.org/10.1007/s00464-014-3766-5

40. Chander J, Vindal A, Lal P, Gupta N, Ramteke VK. Laparoscopic management of cbd stones: An indian experience. Surg Endosc. 2011;25:172-81. https://doi.org/10.1007/s00464-010-1152-5

41. Borie F, Millat B. Laparoscopic treatment of common bile duct stones. Ann Chirurgie 2003;128:722-27. https://doi.org/10.1016/j.anchir.2003.10.029

42. Álvarez LF, Franco AL. Panlitiasis de coledoco: Tratamiento laparoscópico, ¿en qué va la discusión? Rev Colomb Cir. 2000;15:57-61. 
43. Disci E, Atamanalp SS, Ozogul B, Yildirgan MI. The role of choledochoscopy in hepatopancretobiliary diseases. Eurasian J Med. 2016;48:29-32.

https://doi.org/10.5152/eurasianjmed.2015.15299

44. Kotch M, Garden OJ, Padbury R, Rahbari NN, Adam R, Capussotti $\mathrm{L}$, et al. Bile leakage after hepatobiliary and pancreatic surgery: A definition and grading of severity by the International Study Group of Liver Surgery. Surgery. 2011;149:680-8. https://doi.org/10.1016/j.surg.2010.12.002

45. Gómez D, Cabrera LF, Villareal R, Pedraza M, Pulido J, Sánchez S, et al. Laparoscopic common bile duct exploration with primary closure after failed endoscopic retrograde cholangiopancreatography without intraoperative cholangiography: A case series from a referral center in Bogota, Colombia. J Laparoendosc Adv Surg Tech A. 2020;30:267-72. https://doi.org/10.1089/lap.2019.0547

46. Liu J, Jin L, Zhang Z. Laparoscopic Transcystic Treatment Biliary Calculi by Laser Lithotripsy. JSLS. 2016;20:e2016.00068. https://doi.org/10.4293/JSLS.2016.00068

47. Fang CH, Li G, Wang P, Fan YF, Zhong SZ. Computer-aided rigid choledochoscopy lithotripsy for hepatolithiasis. J Surg Res. 2015;195:105-12. https://doi.org/10.1016/j.jss.2015.01.011

48. Sánchez-Ismayel A, Rodriguez O, Salamó O, Pena R, Otaño N, Sánchez R. Uso del láser Holmiun para el manejo de la coledocolitiasis compleja en la explora- ción laparoscópica de las vías biliares. Rev Venez Cir. 2011;64:58-61.

49. Vettoretto N, Arezzo A, Famiglietti F, Cirocchi R, Moja L, Morino M. Laparoscopic-endoscopic rendezvous versus preoperative endoscopic sphincterotomy in people undergoing laparoscopic cholecystectomy for stones in the gallbladder and bile duct. Cochrane Database Syst Rev. 2018(4):CD010507. https://doi.org/10.1002/14651858.CD010507.

50. Alvarado A, Hernández JT, Alvarez A, Chávez A, Lerma $\mathrm{R}$, Arteaga L, et al. Colangiopancreatografia retrógrada endoscópica intraoperatoria como opción segura y eficaz para tratamiento de coledocolitiasis. Cir Endosc. 2016;17:132-8.,

51. Lechaux JP, Lechaux D. Anastomosis biliodigestivas en la litiasis biliar. Técnicas quirúrgicas - Aparato digestivo. EM Consulte. 2008;24:1-9. https://doi.org/10.1016/S1282-9129(08)70149-1.

52. Concepción-Quiñones L, Gutiérrez-Ayala D, Anaya-González JL. Morbilidad y mortalidad de las derivaciones biliodigestivas. Rev Soc Peru Med Interna. 2014;27:68-74.

53. Hirano S, Matsumoto J, Tanaka E, Tsuchikawa T, Shichinohe T, Kato K. Techniques of biliary reconstruction following bile duct resection. J Hepatobiliary Pancreat Sci. 2012;19:203-9. https://doi.org/10.1007/s00534-011-0475-5.

54. Jabłońska B, Lampe P, Olakowski M, Lekstan A, Górka Z. Surgical, treatment of iatrogenic biliary injuries- early complications. Pol J Surg., 2008;80:299-305. 